\title{
Lyme Disease in Maine: a Comparison of NEDSS Surveillance Data and Maine Health Data Organization Hospital Discharge
} Data

\author{
Sara Robinson ${ }^{1}$
}

1. Maine Center for Disease Control and Prevention and University of Illinois at Chicago

\begin{abstract}
Background: Lyme disease is the most commonly reported vector borne disease in the United States and is a major public health concern in Maine. Maine Center for Disease Control and Prevention (Maine CDC) monitors Lyme disease through a passive surveillance system. In order to validate the Lyme disease surveillance system, Maine CDC was interested in comparing trends with a secondary data source. Specifically, Maine CDC was interested in comparing trends by age group, gender, geography, and timelines. Also, because hospitalization due to Lyme disease is rare, this analysis provided an opportunity to look at the diagnosis codes used for Lyme disease visits. The purpose of this paper is to compare the data acquired through surveillance to a secondary data source in order to evaluate the completeness of the data and verify trends. Methods: Surveillance data was extracted from Maine's NEDSS Base System for the years 2008 - 2011. Only confirmed and probable cases were included in data analysis. The Maine Health Data Organization (MHDO) collects information on all hospital inpatient and outpatient data visits and was used for this comparison. MHDO inpatient and outpatient hospital encounters with a diagnosis of 08881 in any diagnosis field were extracted from the full dataset from 2008 - 2011. Results: Surveillance data showed the 5-14 year old age group had the highest rates of Lyme disease while outpatient data showed adults over the age of $\mathbf{4 5}$ to have the highest rates. Outpatient data showed a higher percentage of females with Lyme disease visits. Geographic trends did not match well between surveillance data and MHDO data which may be due to the hospital being used as proxy for the patient address. Timeliness trends were consistent between all sources, with the majority of Lyme disease occurring in the summer months of June, July and August. The majority of outpatient visits had Lyme disease listed as their primary diagnosis while the majority of inpatient visits had Lyme disease as a secondary or lower diagnosis. Conclusions: There were several limitations to this study including incomplete data, and the inability to differentiate between new and old Lyme diagnoses. There is reasonably good similarity in the trends of these two systems helping validate the usefulness of Maine's Lyme disease surveillance system. Many of the discrepancies warrant further investigation, and may lead to future opportunities for education or improvement in Lyme disease surveillance.
\end{abstract}

Correspondence: sara.robinson@maine.gov

DOI: $10.5210 /$ ojphi.v5i3.4990

Copyright (C2014 the author(s)

This is an Open Access article. Authors own copyright of their articles appearing in the Online Journal of Public Health Informatics. Readers may copy articles without permission of the copyright owner(s), as long as the author and OJPHI are acknowledged in the copy and the copy is used for educational, not-for-profit purposes. 


\section{Background}

Lyme disease is the most commonly reported vectorborne illness in the United States, and 95\% of cases are reported from thirteen states, of which Maine is one [1]. Lyme disease is caused by the Borrelia burgdorferi spirochete bacteria and is transmitted through the bite of an infected Ixodes scapularis or deer tick. The most common early symptom of Lyme disease is the formation of a characteristic expanding rash called erythema migrans or EM. Other early symptoms include fever, headache, joint and muscle pains, and fatigue.

In Maine, Lyme disease is the third most commonly reported infectious disease behind Chlamydia and Hepatitis $C^{\mathrm{ii}}$. Cases of Lyme disease have increased exponentially over the last decade following the expansion of the Ixodes scapularis tick [2]. Lyme disease was first detected in the southern counties within the state, but has spread up the coast and into Western Maine. Lyme is now considered endemic in all sixteen Maine counties.

Maine Center for Disease Control and Prevention's Infectious Disease Epidemiology Program is responsible for monitoring disease incidence within the state. Lyme disease is a reportable condition, and Lyme disease surveillance in Maine is a passive system. Maine CDC receives reports of clinically diagnosed Lyme disease (EM rash), as well as positive laboratory results. Each suspect case is entered into Maine's surveillance system which is a NEDSS Base System (NBS). A case report form is sent to the provider to collect information on demographics, symptoms, and risk factors. Returned case report forms are then classified by an epidemiologist using the standard Council of State and Territorial Epidemiologists case definition [3]. The updated information is entered into Maine's NBS. An EM rash alone is confirmatory in Maine, because Lyme disease is endemic in all of our counties.

The Maine Health Data Organization (MHDO) was created by legislature in 1996 to "collect clinical and financial health care information and to exercise responsible stewardship in making this information accessible to the public" [4]. MHDO collects information on inpatient and outpatient hospital encounters which are available annually. This reporting is required in Maine Rules and the definitions of who must submit data and what data must be submitted are clearly spelled out.

Because Maine's Lyme disease surveillance system is a passive system it would be useful to compare the data acquired through surveillance to a secondary source in order to evaluate the completeness of the data and verify trends. Specifically we are looking for similarity in trends of age groups, gender, geography, and timelines. Reports of hospitalizations due to Lyme disease are rare, but we have not reviewed hospitalization records to determine the validity of the rates. Little is known about the individuals hospitalized for Lyme disease in Maine, including if Lyme disease is the primary diagnosis, or a secondary diagnosis.

\section{Methods}

Surveillance data was extracted from Maine's NBS for the years 2008 - 2011. Only confirmed and probable cases were included in data analysis. This data source includes patients who were seen by a provider for Lyme disease and met the federal case definition. 
MHDO inpatient and outpatient hospital encounters with a diagnosis of 08881 in any diagnosis field were extracted from the full dataset from 2008 - 2011. Data were de-duplicated using hospital ID, medical record number, date of service, and sequential visit number. Data for inpatient visits and outpatient visits were analyzed separately. This data includes provider visits for Lyme disease, but no case classification is applied.

All data analysis and manipulation was performed using SAS 9.3 statistical software. All geographic mapping and analysis was performed using Arc GIS Arc Info 10. Rates were calculated using census data for each year, and are per 100,000 persons.

\section{Data and analysis}

\section{Overall results}

From 2008 -2011 data was available for outpatient visits, inpatient visits, surveillance cases, and surveillance cases that were hospitalized. Overall, outpatient visits decreased significantly in 2010 and 2011. Inpatient visits, and surveillance cases that were hospitalized remained relatively stable during all four years. Surveillance cases increased yearly with the exception of 2010 (Table 1).

Table 1: Number of visits for Lyme disease and surveillance cases of Lyme disease - Maine, 2008-2011

\begin{tabular}{|l|l|l|l|l|}
\hline & \multicolumn{3}{|l|}{ MHDO data } & Surveillance data \\
\hline & Outpatient visits & Inpatient visits & Cases & Hospitalizations \\
\hline 2008 & 3048 & 109 & 909 & 38 \\
\hline 2009 & 3544 & 118 & 976 & 46 \\
\hline 2010 & 1173 & 107 & 751 & 25 \\
\hline 2011 & 1278 & 127 & 1012 & 51 \\
\hline
\end{tabular}

\section{Age Groups}

For the purpose of looking at trends by age group, outpatient visit data was used to compare to surveillance data. We assume that inpatient data is likely skewed based on other underlying conditions, so outpatient visit data would be more comparable to surveillance data. The data was stratified into six standardized age groups ( $<5$ years, $5-14$ years, 15 - 24 years, $25-44$ years, $45-64$ years and over 65 years). Counts were converted to rates and are displayed for both outpatient data and surveillance data in Table 2.

Surveillance data consistently shows the highest rate to be in the 5-14 year age group, with the second highest rate in the 45-64 year age group, and the third highest rates in the over 65 years age group. The age group with the lowest rate varies by year (Figure 1). 
Table 2: Rates per 100,000 persons of Lyme disease by age group, surveillance and outpatient data - Maine, 2008-2011

\begin{tabular}{|l|l|l|l|l|l|l|l|l|}
\hline & \multicolumn{2}{|l|}{2008} & \multicolumn{2}{l}{2009} & 2010 & & \multicolumn{2}{l|}{2011} \\
\hline $\begin{array}{l}\text { Age } \\
\text { groups }\end{array}$ & Surveillance & Outpt & Surveillance & Outpt & Surveillance & Outpt & Surveillance & Outpt \\
\hline$<5$ & 43.6 & 63.3 & 58.6 & 88.6 & 36.1 & 21.7 & 68 & 20.7 \\
\hline $5-14$ & 104 & 130.4 & 117.2 & 175.4 & 77.2 & 34.0 & 111 & 46.9 \\
\hline $15-24$ & 50 & 118.6 & 58.2 & 137.6 & 40.6 & 52.5 & 64.4 & 57.8 \\
\hline $25-44$ & 51.6 & 261.2 & 50.8 & 250.0 & 46.2 & 98.8 & 58 & 93.2 \\
\hline $45-64$ & 84 & 304.7 & 85.4 & 352.6 & 65.2 & 113.0 & 85.8 & 130.9 \\
\hline $65+$ & 61 & 255.2 & 69.7 & 356.9 & 59.6 & 114.5 & 71.2 & 121.6 \\
\hline
\end{tabular}

Figure 1: Rate per 100,000 persons by age group, surveillance data - Maine, 2008-2011

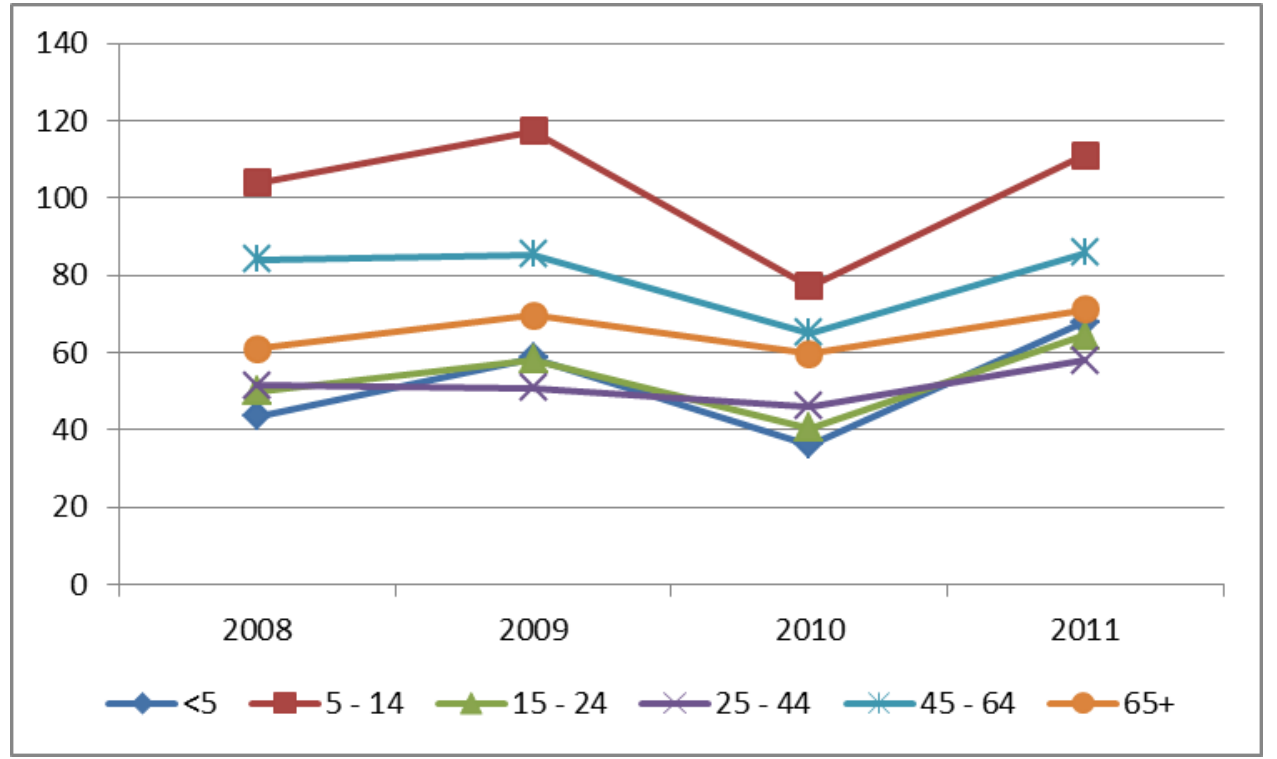

The outpatient data shows the highest rates to be in the $45-64$ years and over 65 years age groups. Children less than 5 years old consistently have the lowest rate in the outpatient data (Figure 2). 
Figure 2: Rate per 100,000 persons by age group, MHDO outpatient data - Maine, 2008-2011

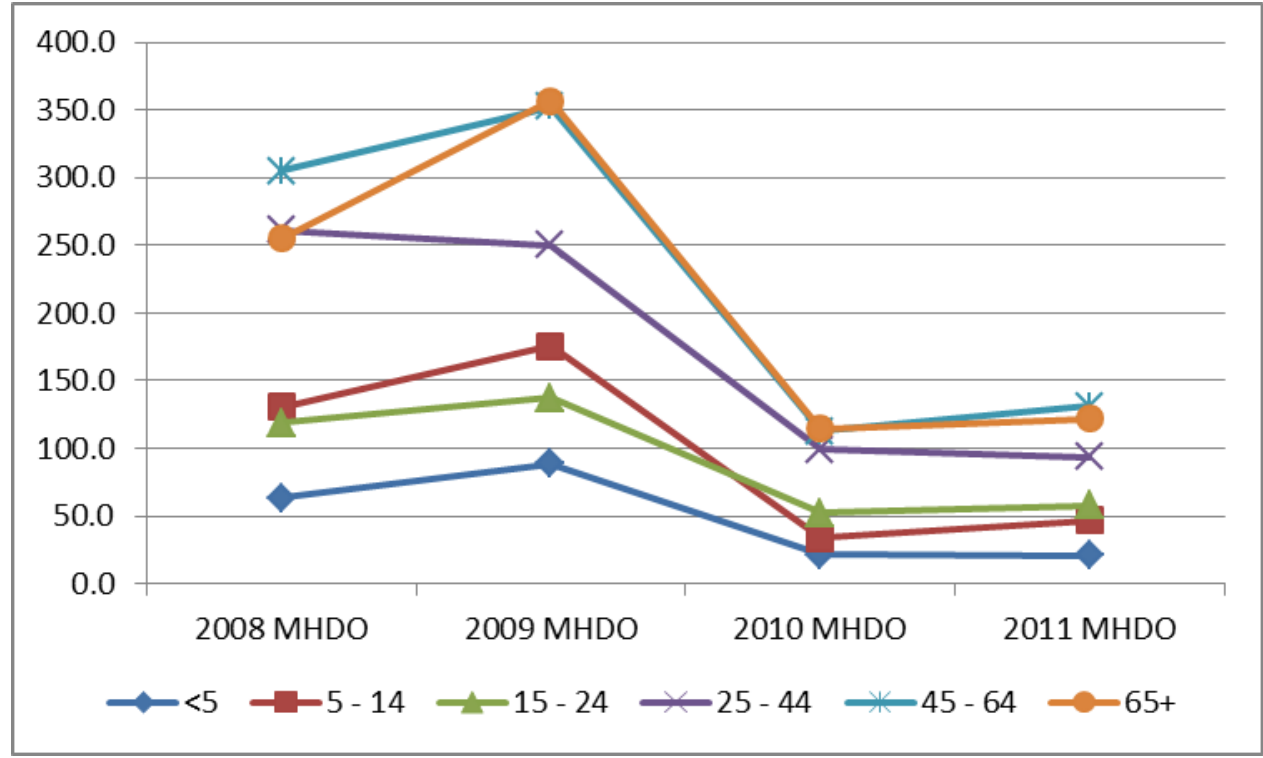

Absolute counts were used to look at hospitalization data because the denominator for hospitalizations was unknown. MHDO inpatient visits were used to due to higher numbers than the surveillance hospitalizations. The majority of inpatient visits for Lyme disease occurred in adults 25 years and older. The age group with the highest count varied by year, but shifted from the $45-64$ to the over 65 years age group over time (Figure 3 ).

Figure 3: Hospitalization by age group, MDHO data - Maine, 2008-2011

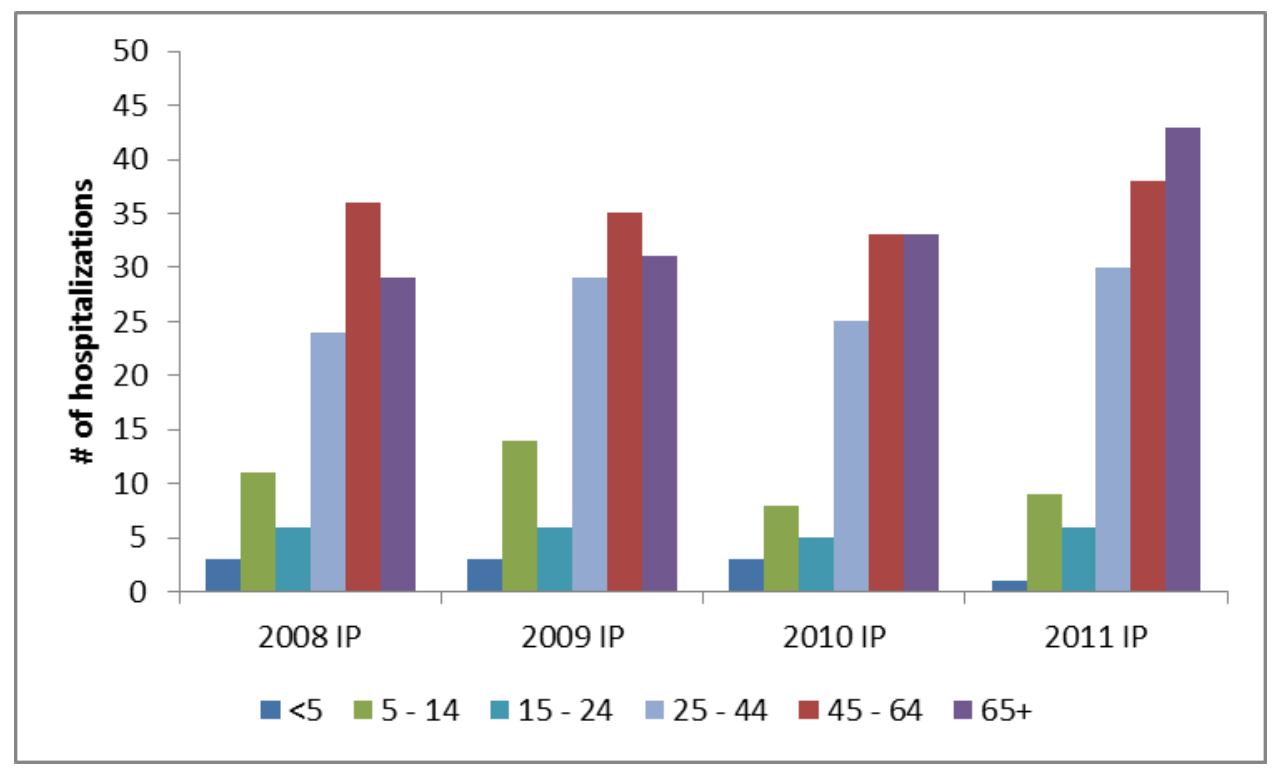




\section{Gender}

Surveillance data and inpatient data showed no significant gender differences for Lyme disease. Outpatient visits had a higher percentage of female visits than male visits; this was consistent for all four years (Table 3).

Table 3: Gender of Lyme disease cases; MDHO and surveillance data - Maine 2008-2011

\begin{tabular}{|c|c|c|c|c|c|c|c|c|c|c|c|c|}
\hline \multirow{3}{*}{$\begin{array}{l} \\
\text { Gende } \\
\text { r }\end{array}$} & \multicolumn{3}{|c|}{2008} & \multicolumn{3}{|c|}{2009} & \multicolumn{3}{|c|}{2010} & \multicolumn{3}{|c|}{2011} \\
\hline & \multicolumn{2}{|c|}{ MHDO } & \multirow{2}{*}{$\begin{array}{l}\text { Surv } \\
\text { Case } \\
\mathrm{s}\end{array}$} & \multicolumn{2}{|c|}{ MHDO } & \multirow{2}{*}{$\begin{array}{l}\text { Surv } \\
\text { Case } \\
\text { s }\end{array}$} & \multicolumn{2}{|c|}{ MHDO } & \multirow{2}{*}{$\begin{array}{l}\text { Surv } \\
\text { Case } \\
\mathrm{s}\end{array}$} & \multicolumn{2}{|c|}{ MHDO } & \multirow{2}{*}{$\begin{array}{l}\text { Surv } \\
\text { Case } \\
\text { S }\end{array}$} \\
\hline & Inpt & $\begin{array}{l}\text { Outp } \\
\mathrm{t}\end{array}$ & & Inpt & $\begin{array}{l}\text { Outp } \\
\mathrm{t}\end{array}$ & & Inpt & $\begin{array}{l}\text { Outp } \\
\mathrm{t}\end{array}$ & & Inpt & $\begin{array}{l}\text { Outp } \\
\mathrm{t}\end{array}$ & \\
\hline $\mathrm{F}$ & 53 & 1805 & 418 & 51 & 1977 & 439 & 50 & 691 & 353 & 55 & 744 & 433 \\
\hline $\mathrm{M}$ & 56 & 1243 & 493 & 67 & 1567 & 531 & 57 & 482 & 398 & 72 & 534 & 579 \\
\hline$\% \mathrm{~F}$ & $\begin{array}{l}48 . \\
6\end{array}$ & 59.2 & 45.9 & $\begin{array}{l}43 . \\
2\end{array}$ & 55.8 & 45.3 & $\begin{array}{l}46 . \\
7\end{array}$ & 58.9 & 47 & $\begin{array}{l}43 . \\
3\end{array}$ & 58.2 & 42.8 \\
\hline
\end{tabular}

\section{Geography}

Surveillance trends in Maine show that Lyme disease was first endemic in the southern counties of the state, and then moved up the mid-coast region. Over half of the Lyme disease cases have occurred in the southern two counties in the state (Cumberland and York). However, these two counties are the most populated counties in the state, so to look at the true burden of disease we converted counts into rates. Accounting for population, the southern two counties (Cumberland and York), and the four Mid-Coast counties (Knox, Lincoln, Sagadahoc, and Waldo) had the highest rates of Lyme disease (Appendix 1). MHDO outpatient visit data rates varied by year. Franklin county in western Maine had consistently high rates, and the Mid-Coast area had relatively high rates. Sagadahoc county had a rate of zero, but this is likely due to the fact that there is no hospital in Sagadahoc county, not that there are no cases there (Appendix 2).

\section{Timelines}

Ticks can be active any time the temperature is above 40 degrees Fahrenheit, so cases of Lyme disease are acquired year round in the state. However, the prevalence of ticks is much higher during the summer months, and therefore we expect to see the majority of the cases to be acquired during these months. Surveillance data uses the onset date to classify the case into months. We used the date of the visit for the MHDO dataset to classify cases into months. The MHDO inpatient and outpatient data and the surveillance data all showed June, July, and August to be the months with the most Lyme disease visits and cases. (Figures 4, 5 and 6) 
Figure 4: Inpatient visits for Lyme disease by month - Maine, 2008-2011

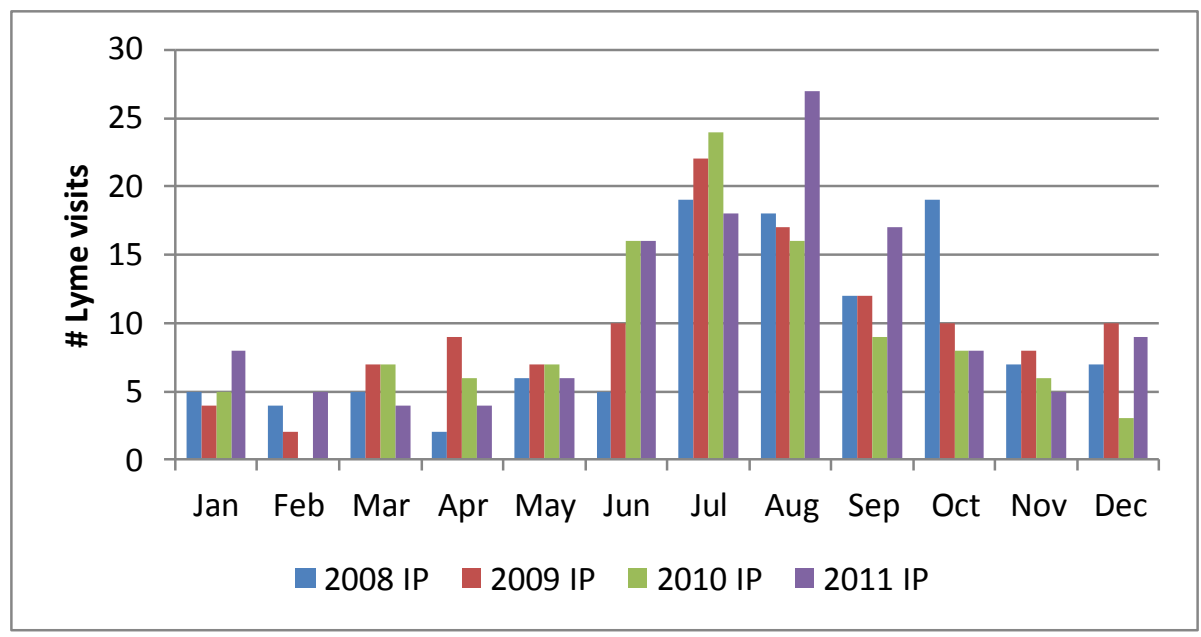

Figure 5: Outpatient visits for Lyme disease by month - Maine, 2008-2011

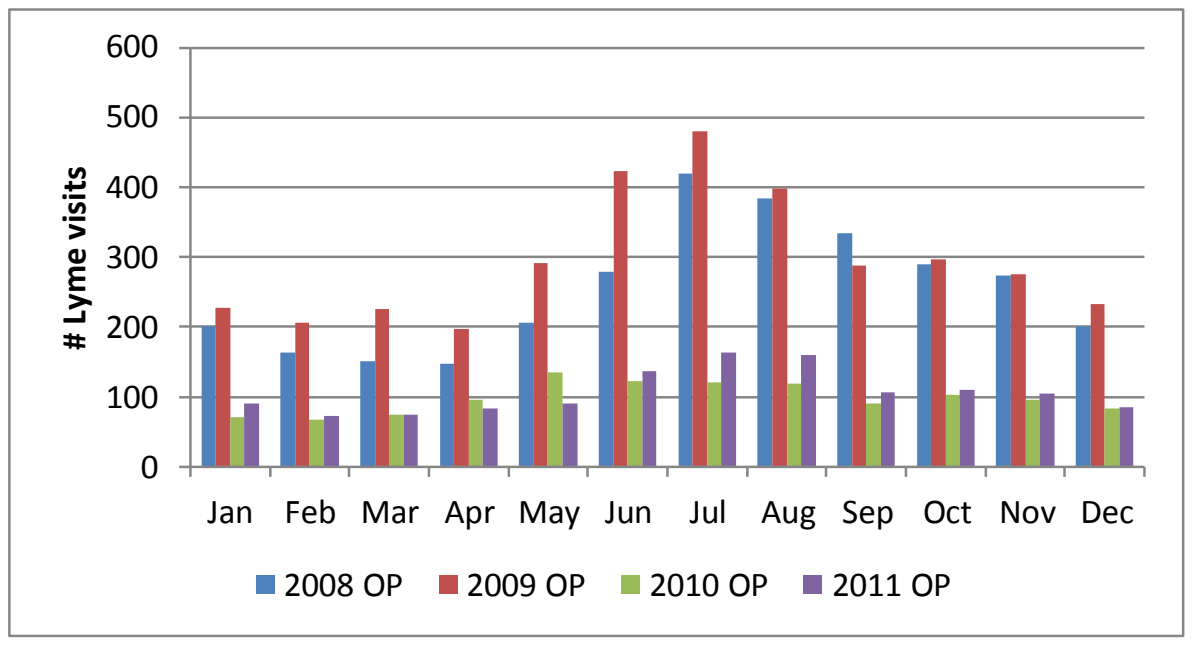

Figure 6: Surveillance cases of Lyme disease by month - Maine, 2008-2011

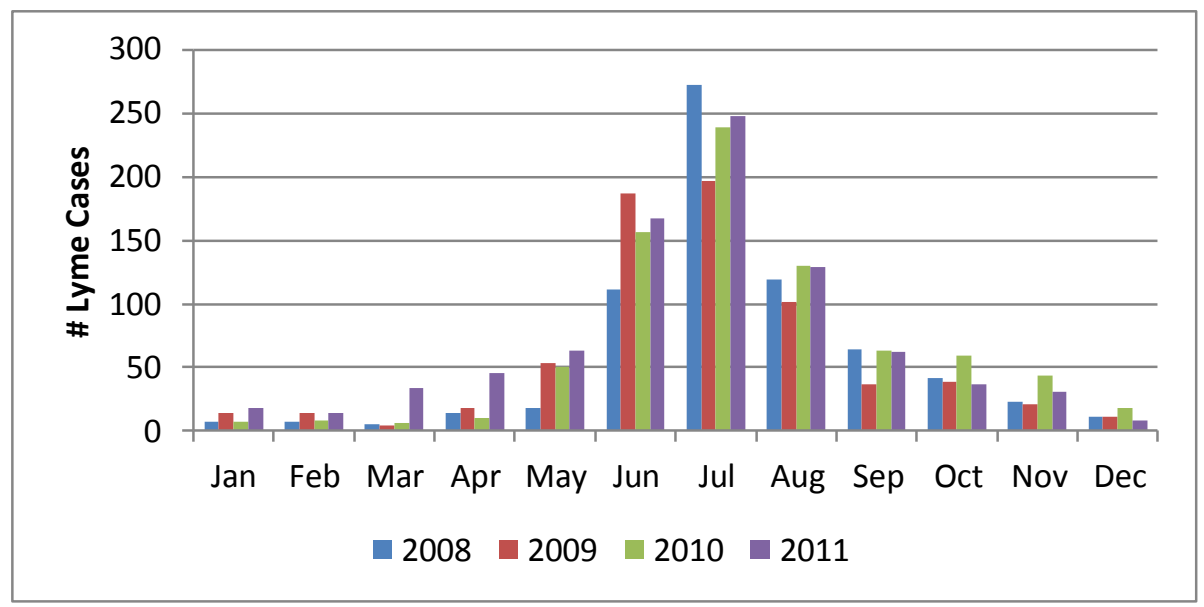

Online Journal of Public Health Informatics * ISSN 1947-2579 * http://ojphi.org * 5(3):e231, 2014 


\section{Diagnosis codes}

The first ten diagnosis codes were available for all MHDO inpatient and outpatient visits. This data was analyzed to determine the percentage of visits with a Lyme disease code within the first five diagnoses. The last five diagnoses were collapsed into an "other diagnosis"category due to the small numbers. It is assumed that Diagnosis 1 is the primary diagnosis; Diagnosis 2 is the secondary diagnosis and so on. Lyme disease was the primary diagnosis in only $27-40 \%$ of inpatient visits (Table 4). More than half of all outpatient visits had Lyme disease as the primary diagnosis (Table 4).

Table 4: Percentage of inpatient and outpatient visits for Lyme disease by diagnosis code Maine, 2008-2011

\begin{tabular}{|l|l|l|l|l|l|l|}
\hline $\begin{array}{l}\text { Inpatien } \\
\mathrm{t}\end{array}$ & $\begin{array}{l}\text { Diagnosis } \\
1\end{array}$ & $\begin{array}{l}\text { Diagnosis } \\
2\end{array}$ & $\begin{array}{l}\text { Diagnosis } \\
3\end{array}$ & $\begin{array}{l}\text { Diagnosis } \\
4\end{array}$ & $\begin{array}{l}\text { Diagnosis } \\
5\end{array}$ & $\begin{array}{l}\text { Other } \\
\text { Diagnoses }\end{array}$ \\
\hline 2008 & 33.0 & 31.2 & 17.4 & 5.5 & 4.6 & 8.3 \\
\hline 2009 & 40.7 & 34.7 & 8.5 & 7.6 & 1.7 & 6.8 \\
\hline 2010 & 27.1 & 36.4 & 17.8 & 7.5 & 3.7 & 7.5 \\
\hline 2011 & 28.3 & 33.1 & 20.5 & 10.2 & 6.3 & 1.6 \\
\hline Outpatient & \multicolumn{5}{|l|}{} \\
\hline 2008 & 71.2 & 15.9 & 7.3 & 2.7 & 1.7 & 1.2 \\
\hline 2009 & 67.9 & 17.4 & 8.8 & 3.5 & 1.3 & 1.1 \\
\hline 2010 & 52.8 & 26.3 & 11.7 & 4.4 & 3.5 & 1.3 \\
\hline 2011 & 52.1 & 22.6 & 11.4 & 6.0 & 4.9 & 3.0 \\
\hline
\end{tabular}

\section{Discussion and Conclusion}

Lyme disease is a major public health concern in Maine. The number of surveillance cases reported each year continues to rise, with no plateau in sight. Because Maine has a passive system for Lyme disease surveillance, it was important to compare surveillance results with a secondary source in order to ascertain the effectiveness of our current surveillance system.

There are several interesting findings through this comparison. This was our first attempt to use MHDO data to look for trends and the MHDO data is not directly comparable to surveillance case data for many reasons. First, individuals may be counted in the MHDO set more than once. Each time the person sought care counted as a new visit, which may dramatically inflate the numbers as one patient may be seen multiple times and at multiple locations. The surveillance data is patient centric, and each individual can only be counted once within a year. It is also recognized that the surveillance case definition is more specific than a clinical diagnosis. In other words, not all clinical cases of Lyme disease will be counted as cases using the surveillance definitions. However, it seems reasonable that although the scale is inflated for the MHDO data, the trends should still exist. The first concern identified in the data is the dramatic decrease in outpatient visits in 2010 and 2011. The surveillance case numbers continued to rise, but the 
outpatient visits fell. This could be due to a change in how the data was collected, or in how the data was pulled for this analysis. This difference warrants further investigation.

In looking at the age group trends, it was surprising to see that the 5-14 year old age group did not have the highest rates in the outpatient data as expected. This could be due to the fact that children may be more likely to see a pediatrician rather than going to a hospital related outpatient clinic, but warrants further investigation. Adults over 45 years had high rates in both the surveillance data and the MHDO outpatient data which was expected. Adults over 45 years also had the highest number of hospitalizations, but this is not surprising because as individuals age they tend to develop other co-morbidities or underlying conditions that may make them more prone to hospitalization in general.

The gender analysis showed a much higher percentage of females with Lyme disease related visits in the MHDO outpatient data than either the inpatient data or the surveillance case data. The literature shows that women tend to access health care more than men, so this may not be an unusual finding [5].

The geographic analysis was perhaps the most interesting finding of this comparison. The rates of Lyme disease cases by county did not match the rates of Lyme disease visits by county. The distribution of surveillance cases matches the distribution of the Ixodes scapularis tick, and is well recognized as how the disease is moving through the state [6]. We expect the MHDO data to be skewed towards population centers because that is where the major hospitals are, and where there are more hospital related clinics. The data are also affected where there is no hospital (Sagadahoc county with a consistent rate of 0 ). However Cumberland county has a consistently lower rate than its surrounding counties which does not make sense. Perhaps there is a difference in how people in different counties utilize the health care system. Maybe some of the larger counties have more options for health care and so they are less likely to use a hospital system provider. It is unclear as to why the geographic trends do not match, but this is definitely something to investigate further.

The timeliness trends showed no surprises. The majority of visits and onset dates for Lyme disease were in the summer months which are when the tick density is highest, but cases are seen year round.

Analysis of the diagnosis data provided some interesting information. The majority of outpatient visits had Lyme as the primary diagnosis, which can be used to infer that Lyme is the reason the patient went to the provider. However, only a small number of inpatient visits had Lyme disease as the primary diagnosis. This suggests that Lyme is either a secondary finding after hospitalization, or a contributing factor in most cases. We expect low hospitalization rates due to Lyme disease itself, so this finding confirms that theory. It would be interesting to see what the common primary diagnoses are for patients for whom Lyme is not the primary diagnosis.

Overall, this analysis showed that there are strengths and opportunities for Maine's current surveillance system. The majority of the trends investigated were similar in both systems which supports the usefulness of the surveillance system. However, the MHDO data has much higher numbers than the surveillance data. It is difficult to tell if this is due to duplication in the MHDO dataset, or missed cases in the surveillance data - it is probably a combination of both. We are 
well aware that providers have low compliance in reporting EM rash alone, and the MHDO data supports the idea that we might be missing clinically diagnosed cases. Further investigation is needed to look at the differences identified through this analysis.

\section{Limitations}

There are many limitations to this analysis. The MHDO data is not as clean as surveillance data, and may be misleading due to multiple visits by a single patient. The data set used for this analysis may be incomplete as suggested by the drop in numbers for 2010 and 2011. Using the facility for the geographic analysis is not as accurate as using patient demographic data, but patient level data was unavailable for this analysis. Another limitation is that the MHDO only contains data for hospital systems and not all outpatient providers. This skews the analysis to those who use emergency rooms and urgent cares as opposed to individuals who may use an independent health care provider. A major limitation is the use of ICD-9 codes for analysis. There is only a single ICD-9 code for Lyme disease, and because of this we cannot tell if the patient is being newly diagnosed with Lyme disease, or if the diagnosis is old. ICD-10 coding will improve this, as it will have multiple coding options for Lyme disease.

\section{Future Directions}

This analysis created some questions that should be investigated further. An investigation into why the outpatient Lyme disease visits dropped so dramatically in 2010 and 2011 is warranted. The geographic analysis should be repeated with patient level demographics as opposed to facility locations to see if that improves the degree of matching between the two systems. Further investigation into the primary diagnoses of inpatient Lyme disease visits may reveal useful information.

As hospital systems begin to change to ICD-10 codes this presents an opportunity to use the Maine Health Information Exchange (HIN) to identify cases that may be missed during surveillance. ICD-10 has a code specifically for erythema migrans, which is confirmatory in Maine. As hospitals begin to switch to ICD-10 codes Maine CDC can collaborate with Maine HIN to transmit cases with this diagnosis directly to our NBS. This will improve provider compliance with reporting and will make our surveillance system more robust.

\section{References:}

1. Centers for Disease Control and Prevention. Lyme Disease. Updated December 6, 2013. Available at http://www.cdc.gov/lyme/stats/index.html. Accessed 12/10/2013.

2. Robbins A, Mallis H. Reportable Infectious Disease in Maine: 2012 Summary. Available at http://www.maine.gov/dhhs/mecdc/infectious-disease/epi/publications/2012-annual-report.pdf. Accessed $12 / 10 / 2013$.

3. Centers for Disease Control and Prevention. National Notifiable Diseases Surveillance System (NNDSS): Lyme. Updated November 14, 2013. Available at http://wwwn.cdc.gov/NNDSS/script/conditionsummary.aspx?CondID=100. Accessed 12/10/2013.

4. Maine Health Data Organization. Available at https://mhdo.maine.gov/index.aspx. Accessed 11/10/2013.

5. Bertakis KD, Azari R, Helms LJ, Callahan EJ, Robbins JA. 2000. Gender differences in the utilization of health care services. J Fam Pract. 49(2), 147-52. PubMed 
6. Maine Medical Center Research Institute. Lyme and Other Vector-borne Disease Information: Map of Deer Tick Distribution in Maine Updated 2011. Available at http://www.mmcri.org/home/webSubContent.php?list=websubcontentlive\&id=197\&catID=4\&subCatID=19. Accessed 12/10/2013. 
Appendix 1: Surveillance Lyme Disease Rates by county, Maine 2008-2011

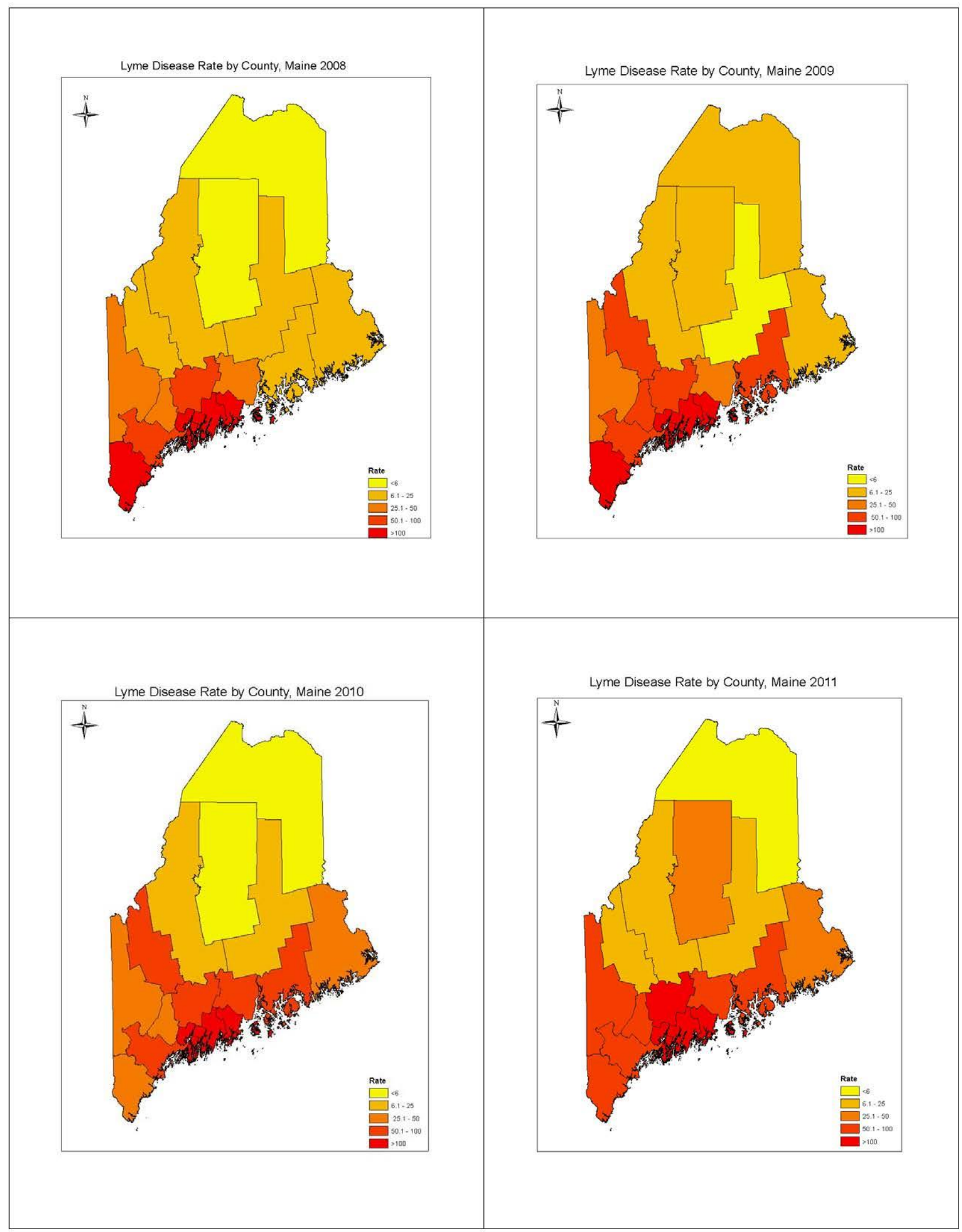

Online Journal of Public Health Informatics * ISSN 1947-2579 * http://ojphi.org * 5(3):e231, 2014 
Appendix 2: MHDO Outpatient Lyme Disease Rates by county, Maine 2008-2011

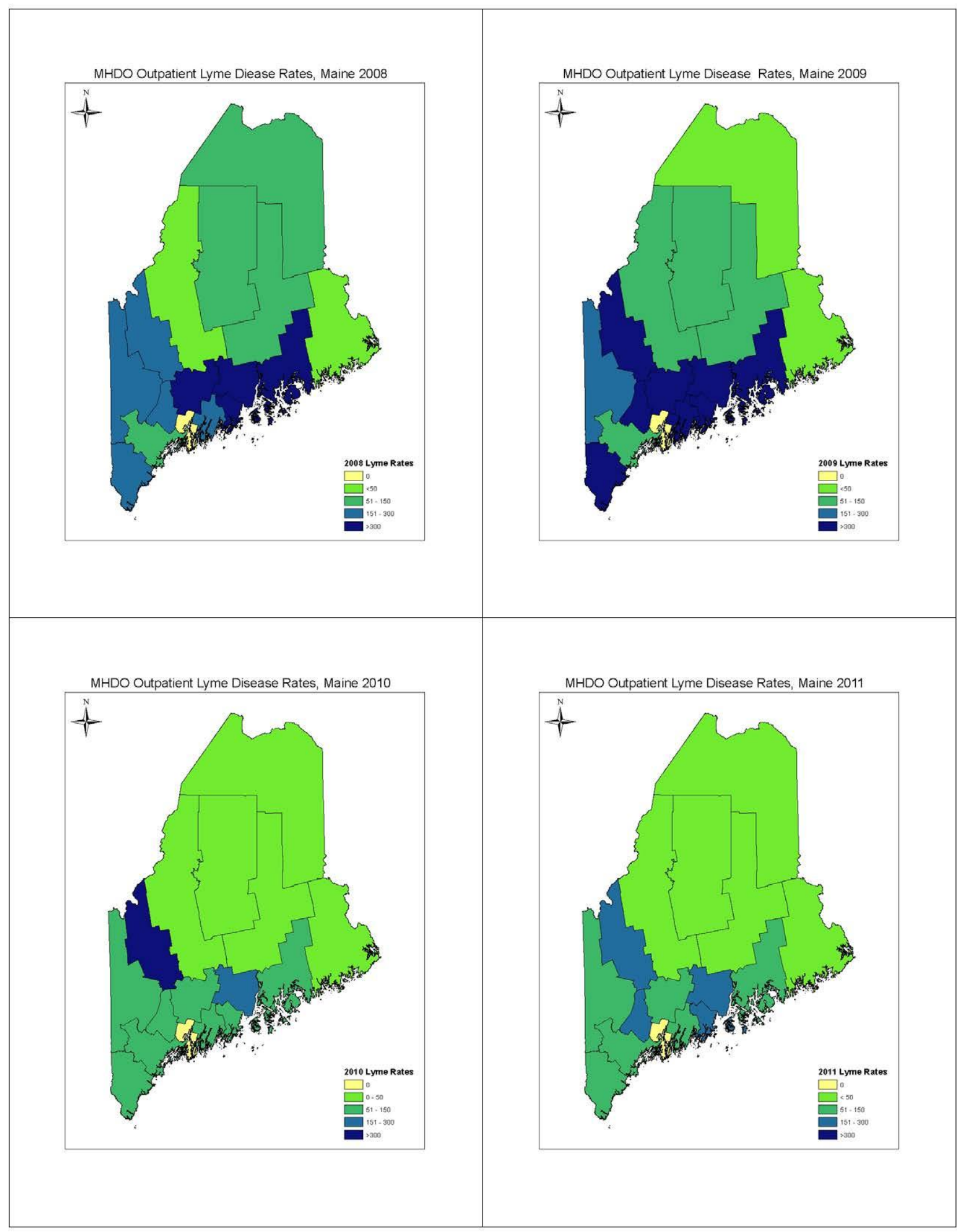

